\title{
Technology of swallowable capsule for medical applications
}

\author{
Ioannis Intzes, Hongying Meng* and John Cosmas \\ Department of Electronic and Computer Engineering \\ Brunel University, \\ Kingston Lane, Uxbridge \\ Middlesex UB8 3PH \\ E-mail: ioannis.intzes@,brunel.ac.uk \\ E-mail: hongying.meng@brunel.ac.uk \\ E-mail: john.cosmas@brunel.ac.uk \\ *Corresponding Author
}

\begin{abstract}
Medical technology has undergone major breakthroughs in recent years, especially in the area of the examination tools for diagnostic purposes. This paper reviews the swallowable capsule technology in the examination of the gastrointestinal system for various diseases. The wireless camera pill has created a more advanced method than many traditional examination methods for the diagnosis of gastrointestinal diseases such as gastroscopy by the use of an endoscope. After years of great innovation, commercial swallowable pills have been produced and applied in clinical practice. These pills can cover the examination of the gastrointestinal system and not only provide to the physicians a lot more useful data that is not available from the traditional methods, but also eliminates the use of the painful endoscopy procedure. In this paper, the key state-ofthe-art technologies in the existing wireless capsule endoscopy (WCE) systems are fully reported and the recent research progresses related to these technologies are reviewed. The paper ends by further discussion on the current technical bottlenecks and future research in this area.
\end{abstract}

Keywords: ASIC, FPGA, NFC, SmartPill, wireless capsule endoscopy, swallowable devices, wireless capsule endoscopy capsule (WCE).

Reference to this paper should be made as follows: Intzes, I., Meng, H., and Cosmas J. (2014) 'Technology of swallowable capsule for medical applications', Int. J. Biomedical Engineering and Technology, Vol. x, No. x, pp.xx-xx.

Biographical notes: Ioannis Intzes is a $\mathrm{PhD}$ student at Electronic and Computer Engineering Department, Brunel University, UK obtained his BEng in Electronic Engineering from the Technological Educational Institute of Thessaloniki, Department of Electronics in 2008. He completed his MSc in Wireless Communication Systems from the Brunel University in 2012.

Hongying Meng is a lecturer in multimedia communication and embedded systems at Electronic and Computer Engineering Department, Brunel University, UK received Ph.D. degree in Communication and Electronic Systems from Xi'an Jiaotong University in China. He has a wide research interests including digital signal processing, machine learning, human computer interaction, computer vision, image processing and embedded systems. 
John Cosmas is a professor in multimedia systems at Electronic and Computer Engineering Department, Brunel University, UK received his BEng in Electronic Engineering at Liverpool University, UK in 1978 and a PhD in Image Processing at Imperial College, University of London, UK in 1986. His research interests are concerned with the development and application of multimedia communications systems. Prof. Cosmas currently serves as associate editor for IEEE Trans. Broadcasting.

\section{Introduction}

Wireless capsule endoscopy (WCE) is an advanced technology for medical diagnoses of gastrointestinal diseases and illnesses. The idea was originally started in 1950 when a lot of research effort had been done to improve the diagnoses abilities using new technologies. Through the years, WCE has been given different names like, smartpill, wireless endoscopy, video capsule, etc. The very first attempts of WCEs, use transmitters with low frequency carrier and the diagnosis was based only from sensor's data like temperature, $\mathrm{pH}$, and pressure. With the technology evolution, WCEs could support more accurate diagnosis by combining small cameras with low resolution and the sensors' data.

For medical practice, capsule endoscopy was, introduced commercially by the Israeli company GivenImage, Inc. at 2009. The first capsules were designed for investigating the small bowel because it was hard to investigate with the traditional techniques. These commercial products were able to provide low resolution images at low frame rate. Nowadays there are capsules dedicated for specific investigation areas, such as esophagus (PillCam ESO) and colon (PillCam COLON). The most recent product of GivenImage, contains two cameras, (PillCam COLON) horizontally placed in the pill, one in the front of the pill and one in the back, and it can support up to 30 frames per second.

The academic/scientific community has made significant contribution in the evolution of this technology and has come up with interesting products and ideas. The most common diseases that WCEs can diagnose are some types of cancer, Crohn's disease and obscure gastrointestinal bleeding. By the use of WCE, tissue can be taken from specific areas for biopsy.

The current WCE products can be categorized in different ways. The pills can be categorized by their function as drug delivery capsule, sensor capsule, camera capsule and the most hardware complicated category, robotic capsule which has parts for locomotion and electromechanical actuators for biopsy.

In the drug delivery category, the pill is composed with a "drug delivery" system and a couple of sensors. The pill is programmed in such a way that it can deliver the drug due to sensors' data in the Gastrointestinal (GI). In category of sensor capsule, the pill uses sensors and a wireless system to transmit the measurements to a host computer for further processing. The most common sensors are temperature, $\mathrm{pH}$, pressure and oxygen. The camera capsule category, as the title says, contains one or more cameras and with the use of wireless communications schemes, transmits the images/video to a host computer. Most of them have the ability for real time image/video representation. The robotic capsule category is the most complex one that combines a lot of parts from the other three categories and has moving mechanical parts.

The camera capsule has been gained more interests from researchers due to the evolution of technology on tiny size of cameras and microprocessors. Extensive research has been done to design and improve the existing systems to the next level. 
In the rest of the paper, the key technologies in the existing wireless capsule endoscopy (WCE) systems are introduced in section 2. The state of the art research progresses in these technologies is reviewed in section 3 . The paper ends by discussions on the current technical bottlenecks and further research in this area in section 4.

\section{Key technologies in WCEs}

The endoscopic capsules play a major role in the diagnosis of the gastrointestinal (GI). The traditional methods are painful for patients and is not possible to examine the entire gastrointestinal (GI). In order to achieve the high demands of medical diagnosis on the entire gastrointestinal track, higher performance wireless capsule must be used.

A wireless capsule, despite its size, consists of a lot of electronic parts. There are sensors, the processing unit, the wireless transmission unit and the power management unit, which is usually integrated with the processing unit.

In the part of the sensors, as mentioned before, various types are used like, pressure sensor, temperature sensor, inertial sensor, $\mathrm{pH}$ sensor, conductivity sensor and oxygenation sensor. For example with a $\mathrm{pH}$ sensor the detection of acidic reflux can be diagnosed, or with the electrical conductivity sensor the detection of non-acidic reflux can be diagnosed as shown in Figure 1. Both of these measurements can provide more information, for the final diagnoses, to the physicians.

\subsection{Sensors and vision equipment}

Normally every capsule contains one kind of sensor and can take measurements for up to 24 hours, like VitalSense ingestible telemetric physiological monitoring system, which is a pill that uses thermistor to measure the temperature as shown in Figure 2. Another way to measure the temperature is the way that the CorTemppill system utilizes. It uses Figure 1

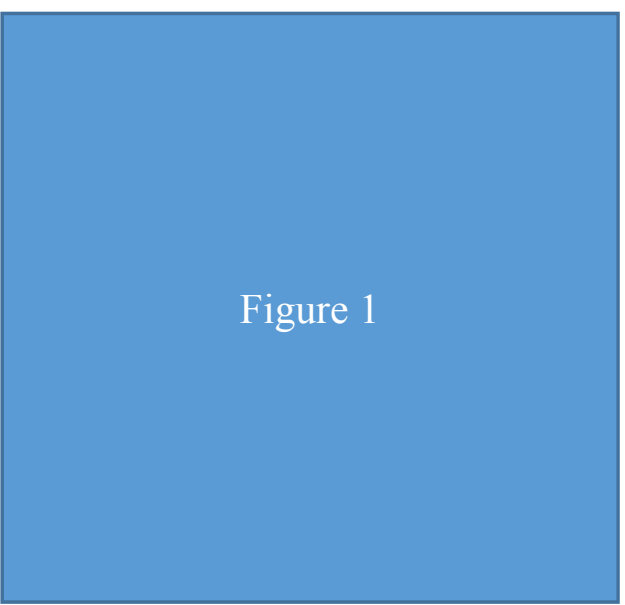
the frequency oscillation of a crystal to sense the temperature. However there are capsules that contain more than one type of sensor. The Lab-on-a-Pill capsule is a capsule that can sense $\mathrm{pH}$, conductivity, and oxygen levels. The abilities of these biosensors can provide useful information for the physicians. Apart from these sensors new techniques are researched for specific uses, like biopsy. By the use of light the WCE can perform an optical biopsy without the need of extraction of tissue samples, which in some cases may be impossible. 
Camera can be regarded as an image sensor. The pills that uses camera to diagnose are using state of art technology for capture, compression and transmission circuits. Just like the previous sensor, the camera's characteristics are dependent on the investigating area, such as esophagus (PillCam ESO) and colon (PillCam COLON). For this sensor, two different ways of camera placement are observed, vertically to the pill and horizontally. In Figure 3, is an example of horizontal placement from GivenImagine, Inc. In Figure 4, an example of a vertical placement of the camera in the SAYAKA pill from RF System Lab.
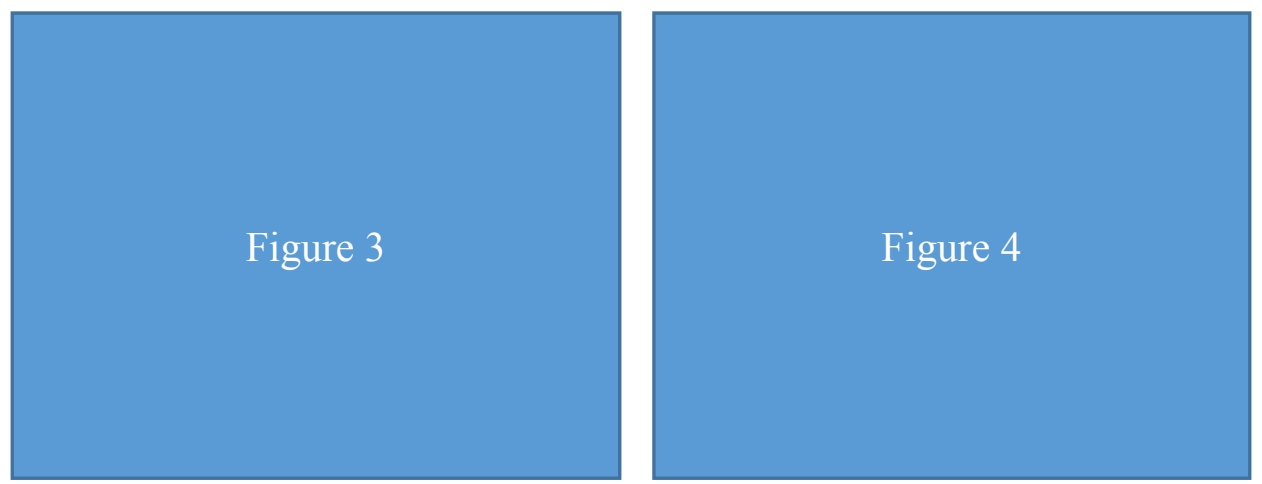

Due to the camera's placement and an electromechanical system, that can rotate the camera, SAYAKA pill can take 3600 photos. The camera module has a resolution of 2 mega pixels and a frame rate of $30 \mathrm{fps}$, a full rotation of the camera takes about 12 seconds. The bottleneck of this system is the unstable speed of the capsule. Due to this problem, approximately the $70 \%$ of the gastrointestinal (GI) can be captured. Pills like this, with vertical placement of the camera, are used in the mapping of the entire gastrointestinal (GI) with high resolution images. To accommodate different functional requirements, the pills have different resolutions and frame rates. For example the Olympus pill has an HD camera module with a resolution of $1920 \times 1080$ pixels which is horizontally placed in the capsule and takes 2 frames per second. On the other hand, GivenImagin's pill takes lower resolution images but at a higher frame rate. Table 1 is the summary of the most known WCEs from companies and universities. Two types of camera sensor are used, namely: CCD and CMOS. Most of the existing systems of camera pills are using CMOS technology camera sensor, except of Olympus EndoCapsule, that uses CCD. In the next table, the most known camera pills and their camera characteristics are listed.

The resolution of the camera is not the only criterion for obtaining the best images/video. In the part of a camera module the optic lenses can take a serious 
advantage in the final quality of the image/video. All the commercial products mentioned before have lenses with static angle of view. There is research in liquid lens and their electric control so the adjustment of the focal length can happen via electronics.

\subsection{Processing Unit}

All these sensors need control, so a process unit is used. It is the heart of the capsule that can handles all the data from the sensors. It converts the analog signals of the sensors to digital for further processing, also takes huge amount of data of the camera and compresses them so a low data rate wireless link can be used. A state of the art development system of capsule endoscopy has been created and can achieve QVGA resolution images at $19.53 \mathrm{fps}$, the

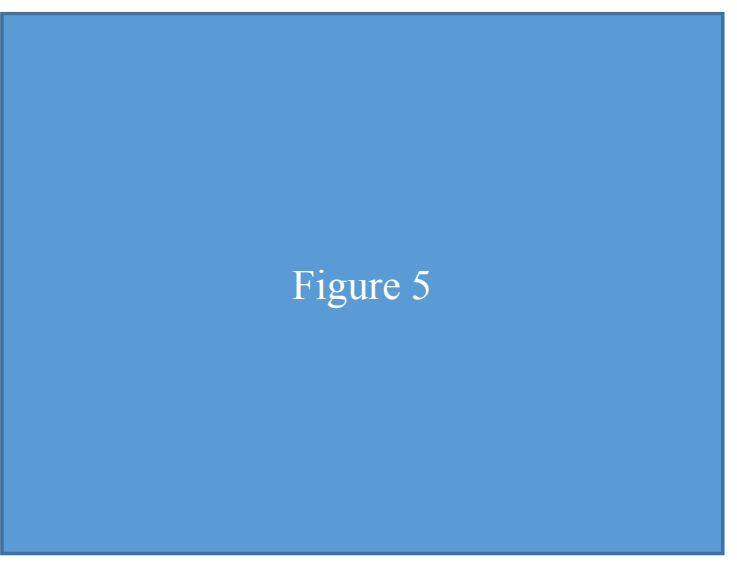
power consumption of this system is only $26 \mathrm{~mA} @ 1.8 \mathrm{~V}$. At Figure 5 a prototype of this system is shown. It is obvious that the need of a high performance and low power consumption system is needed. The process unit must have low power consumption but at the same time it must handle all these data in a too small time. A novel design of a process unit, as we mentioned, has been made and has interesting characteristics. A VLSI design architecture of three-stage clock management is applied, so a power saving of up to $46 \%$ is obtained, compared to designs without this low-power technique. The main characteristics of this capsule design are the image resolution of 320x288 pixels at $8 \mathrm{fps}$ and transmitted with a wireless link of $2 \mathrm{Mbps}$ bandwidth. The process unit has been implemented in $0.18-\mathrm{m}$ CMOS process and it consumes only $6.2 \mathrm{~mW}$ (only the process unit).

There are still a lot of challenges in the sensors inside. In the sensors of temp, $\mathrm{pH}$, oxygen, conductivity, etc. the challenges resulting, are in the domain of the size mostly and in the accuracy. These types of sensors don't need high sampling rate because the pill is moving too slow. But in an image sensor, or cameras, there are a lot more challenges that researchers need to provide a solution. For example, in these systems the need of clear and sharp images is obvious. So, a small camera with great characteristics (resolution, FPS, etc.) and optics is the area that needs to be improved. With high quality images, better diagnosis can be achieved.

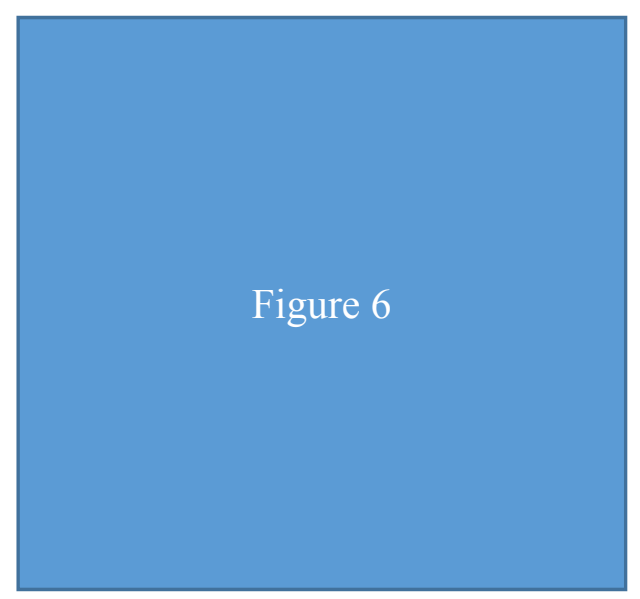

\subsection{Wireless transmission}

The ability of the capsule to achieve high quality diagnostic results mostly relies on the wireless communication link and its characteristics. 
Endoscopic capsules have some characteristics which are unchangeable, like its size. It is obvious that with this small size the energy that can be stored, is too low. So, the power consumption is a big criterion for the design of the whole system and especially of the wireless link. The circuit for wireless transmission can be made by commercially available chips but in most cases the utilization of a custom solution is used due to the small size needed. The wireless link used

in wireless pills can be categorized into two categories, the bidirectional and unidirectional. In most cases the use of a unidirectional link is implemented, since the reason is that the capsules are only transmitting the compressed data of the sensor/camera to the external device for further processing. A unidirectional wireless link proposed by Thoni et al in Figure 6 is a novel design of a simple topology FSK modulation transmitter that can achieve up to $2 \mathrm{Mbps}$ of data rate and power consumption to as low as $2 \mathrm{~mW}$ at $1.8 \mathrm{~V}$. This was an example of a wireless unidirectional circuit used on the other hand there are also system with bidirectional links. The reason for using a bidirectional link is that there are some robot capsules that need to receive commands from outside the patients' body. A design of a unidirectional wireless link has been made and has great characteristics as shown in Figure 7. It has an uplink up to 10Mbps and a downlink of $10 \mathrm{Mbps}$. Downlink and uplink are both configurable from $1 \mathrm{Mbps}$ to $10 \mathrm{Mbps}$. It works in Ultra Wide Band (UWB) frequency area at $3-5 \mathrm{GHz}$. Both transmitter and receiver consume only $10 \mathrm{~mW}$ with a work cycle of $80-100 \%$ and $1-80 \%$ respectively.

Due to the small data rates that the existing systems have, the resolution of the images is not high and they operate at a low frame rate $2-4$ fps. So the design of a wireless link with high data rates and with low power consumption can improve the performance of the whole system. In the table 2, a few high performance designs are compared.

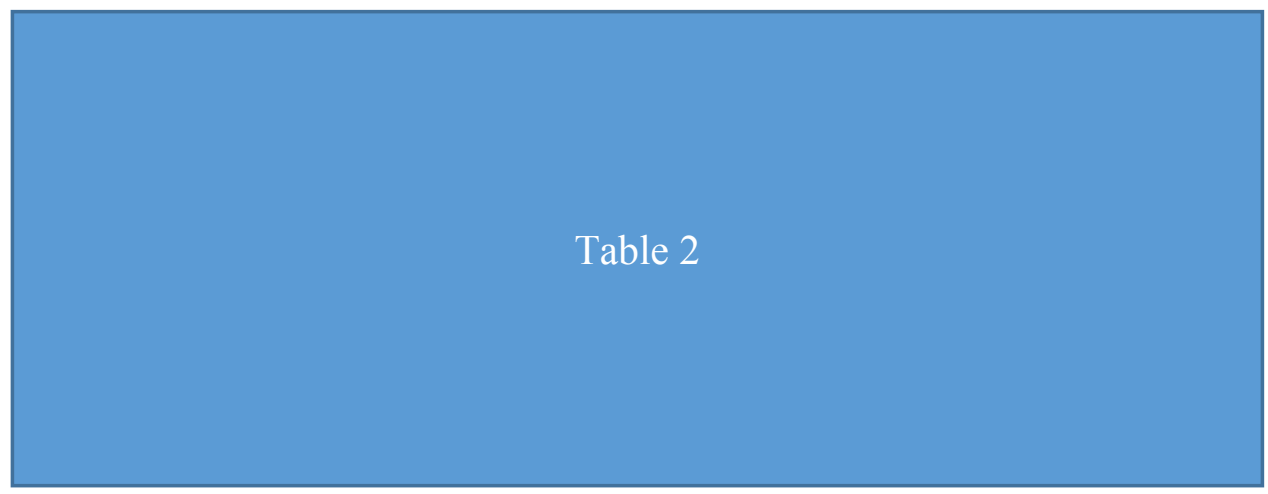

\subsection{Power stage}




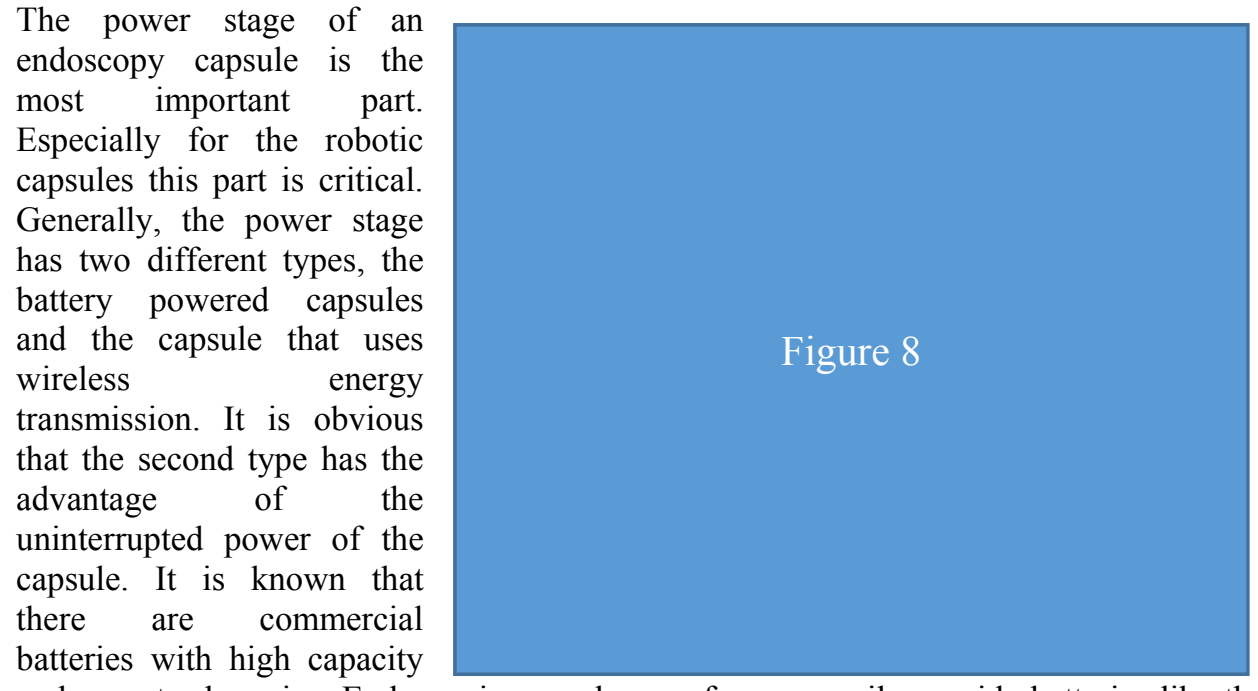
and an extra low size. Endoscopic capsules use for power silver-oxide batteries like the ones watches use. This type of battery is approved for clinical use. With this kind of batteries, it is impossible to power a robotic capsule, since they can only be used for sensor based capsules.

The category without battery can provide the capsule with much more energy but the system for energy harvesting is bigger than a battery. Figure 8 shows such systems and comparison with the size of 1-cent coin. A system with high energy consumption is a 12-legged robotic capsule and consumes up to $300 \mathrm{~mW}$ of energy! It is obvious that in such a system, it is not possible to use only batteries to power it.

It is clear that in system with low energy consumption, battery is the best solution. But in robotic capsules system, the use of wireless energy transfer is more suitable. An inductive power transmission has been developed and can deliver up to $150 \mathrm{~mW}$. This system is designed to work at $1 \mathrm{MHz}$ and has an efficiency of about $90 \%$. The selection of this frequency was due to the restriction of the SAR, so with this frequency, the absorption rate is kept down to $0.4 \mathrm{~W} / \mathrm{Kg}$. The whole system can fit in $10 \mathrm{~mm} \varnothing$ and $13 \mathrm{~mm}$ length.

In previous paragraphs sensors/cameras, process unit, wireless and power system are discussed. These parts are frequently used in the endoscopic capsules. There are also some types of capsules that use three different systems, localization, locomotion and intervention \& tissue manipulation systems. All of them are used in robotic capsules except of the localization system that some sensor capsules are using.

\section{$2.5 \quad$ Localization}

To determine where the images/video is taken or where the robotic capsule has to operate, localization systems has been researched and developed. These systems give the ability to the physician to have

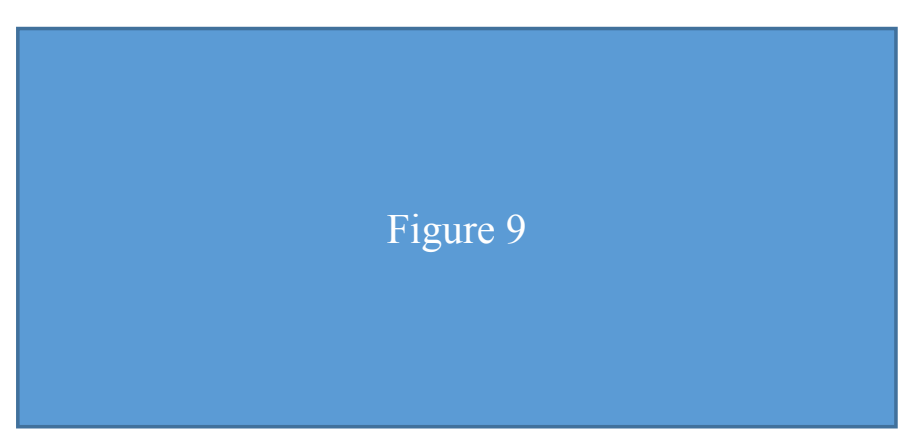


a clear view of the problem when they are looking at an image which is captured from the capsule. It helps to know the distance travelled so an intersection or the diagnosis can have better results. There are a few ways to locate the capsule in the gastrointestinal (GI). The most known and used is the RF localization. It uses the triangulation of the radio frequency that the capsule transmits to compute the capsule's position. RF localization is used by the commercial product of Israel Company GivenImage M2A capsule. This technique is commonly used due to the fact that it needs only external sensors to find the location of the capsule and is not expensive. A work in localization systems reported in as shown in Figure 9, found that by the use of 32 external sensors, 16 in front of the patient and 16 in the back of the patient great accuracy can be achieved. The system is working at $402-405 \mathrm{MHz}$.

Human gastrointestinal track is a challenging environment for investigation. It has the potentiality to move into the abdominal cavity and this means that is difficult to know where the pill is located. This problem can reduce the opportunity of a physician to make a diagnosis. This system, by providing the location of the pill, may make the captured image more understandable. As we mentioned the gastrointestinal (GI) is moving into the abdominal cavity so there are many curves and fewer straights surfaces and some images are not clear enough, so without the location of the pill it is too hard to understand a possible disease. It is obvious that the localization system can take a major role into the diagnosis.

\subsection{Locomotion}

The movement of the capsule is made by the movement of the gastrointestinal (GI), named peristalsis. There are typically two main categories of locomotion, the active and the passive. Peristalsis is a passive way which doesn't need any electronic circuits or special mechanical design of the capsule. The active category has obtained a lot of interest. In passive locomotion, a physician is not able to control the movement of the capsule. Due to this problem, critical images will not be captured and the diagnosis will be incomplete. By the use of active locomotion the physician can "drive" the capsule and take more images, which are probably clearer, at the areas of interest. There are two categories of active propulsion methods, internal and external. The internal method uses micro mechanisms to move the capsule and the external uses external forces like magnetic field.

Internal method of locomotion uses state of the art electrical and mechanical design to work and has been used in current products. Capsule with internal locomotion uses
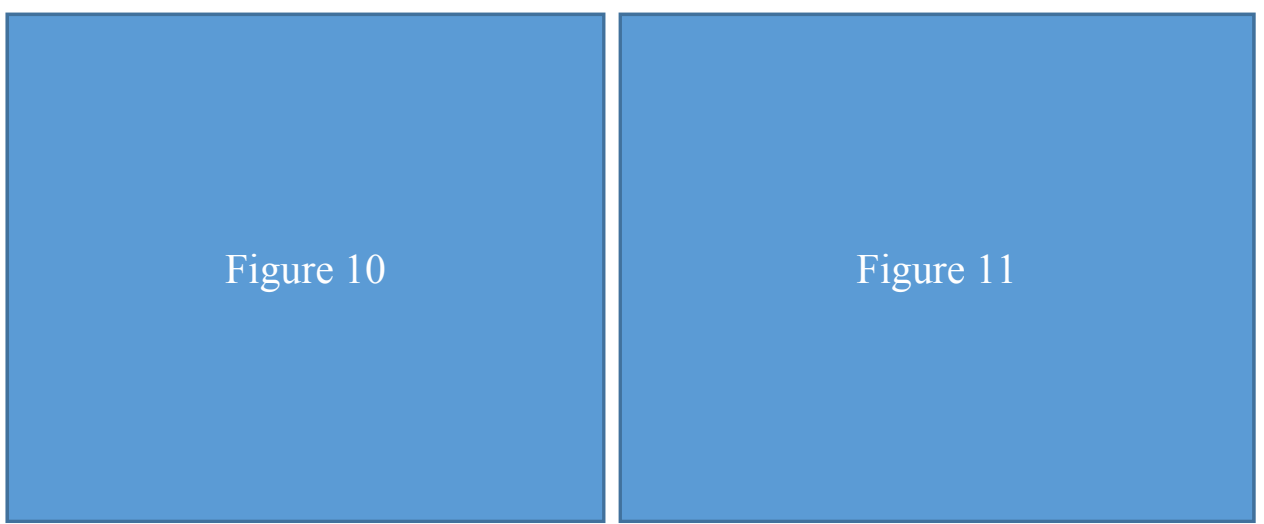
"legs" for locomotion as shown in Figure 10. The main advantage of this technique is the physical contact and the movement can be more precise. There is a lot of research effort in internal locomotion techniques. Another technique of locomotion is proposed and it uses special alloys that have shape memory. This means that they use this special function of these alloys to make movement. There are various ways to create movement. Another approach is the propelled capsule, which is using a propeller to give a boost in the movement of the capsule.

\section{Recent progress on key technologies in WCEs}

Recent years, more and more researches have been done in order to improve the performance of current WCE products. In the following, we will review the recent research progress on the key technologies in WCEs such as image sensors, wireless communication, power consumption, external locomotion and image processing.

\subsection{Image sensor and image compression:}

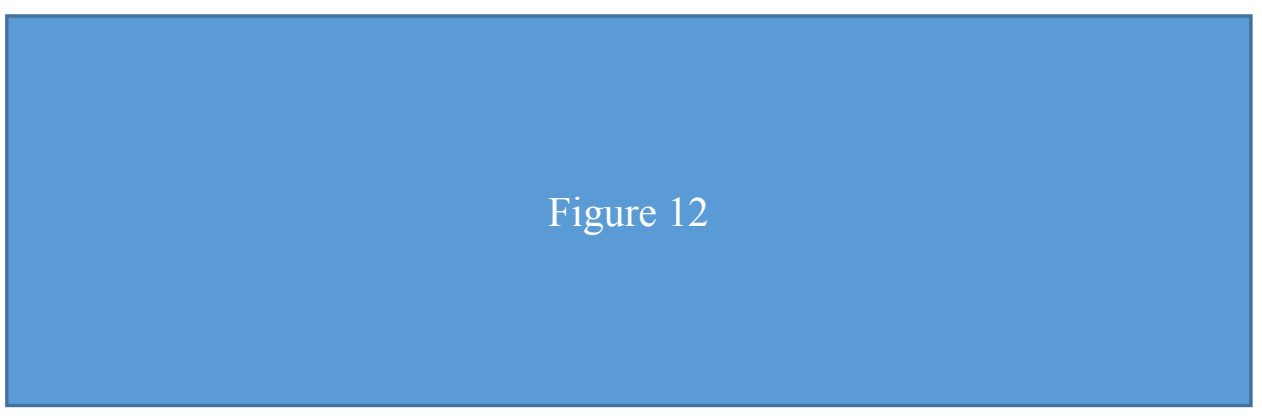

There are a lot of investigations on the image sensor with the compression algorithm in a WCE. Turcza and Duplaga proposed a novel design for an entire pill. A custom CMOS sensor with a dedicated image compressor was designed and implemented to provide next generation endoscopic pill. The compression algorithm is based on the integer discrete cosine transform (DCT) with a low complexity, high efficiency, entropy encoder. The entropy encoder uses the adaptive Golomb-Rice algorithm instead of Huffman tables. The image sensor captures $320 \times 240$ pixels per image. Figure 12 shows the compression algorithm procedure.

Shirsat and Bairagi recently proposed a novel method for image compression. This method uses a lossless image compressor which is based on integer wavelet transform (IWT) and predictive coding and achieves a fast and low complexity algorithm. An important factor for wireless capsule endoscopy is the complexity of the compression algorithm because by this way the minimum power consumption can be achieved. It is also remarkable the low demand of memory for processing of image data. This kind of the compression algorithms has significant advantage to be used in future WCEs.

\subsection{Wireless transmission}


Gao et. al has recently proposed an entire capsule system with a very interesting work on wireless communication technology in the WCE. It was implement in $0.18 \mu \mathrm{m} \quad$ CMOS QPSK transmitter that can achieve up to $3 \mathrm{Mb} / \mathrm{s}$ data rate and an OOK receiver with a data rate of $500 \mathrm{~kb} / \mathrm{s}$. The power consumption is $5 \mathrm{~mW}$ at $6 \mathrm{dBm}$ of output power while the receiver has a $-65 \mathrm{dBm}$ sensitivity in $4.5 \mathrm{~mW}$. Figure 13 is a photograph of the RF SoC.

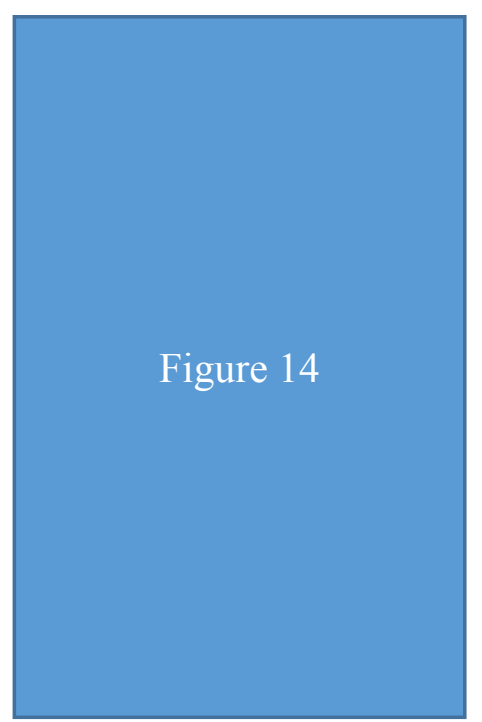

Antenna has a major role in the quality of the wireless part. Recently, Thotahewa et al proposed an excellent work on the design of antennas for the Ultra Wide Band. The use of Impulse Radio-Ultra Wide Band (IR-UWB) technology promises high data rate capabilities with low energy consumption, in small factor. This work simulates the performance of such a system and namely is focused on the Specific Absorption Rate (SAR) and compares the results with the international safety regulations.

Pourhomayoun et al recently proposed a novel design of a wireless system where the capsule localization is mentioned as shown in Figure 14. The use of external sensors can detect the location of the capsule wirelessly. By the use of 32 receiver sensor, the RMS error is around $1.5 \mathrm{~cm}$. The design of this system doesn't have limitations like the WCE because it is outside the human body. Localization systems can help the physicians to extract better diagnosis results.

\subsection{Power consumption}

Power technology has made significant progress in recent years. Sun et al proposes a novel wireless energy transfer method. It is an omnidirectional antenna design. It can effectively combine the wireless energy from all directions. The advantage of this method is that the energy reception is from any direction. It can achieve greater overall

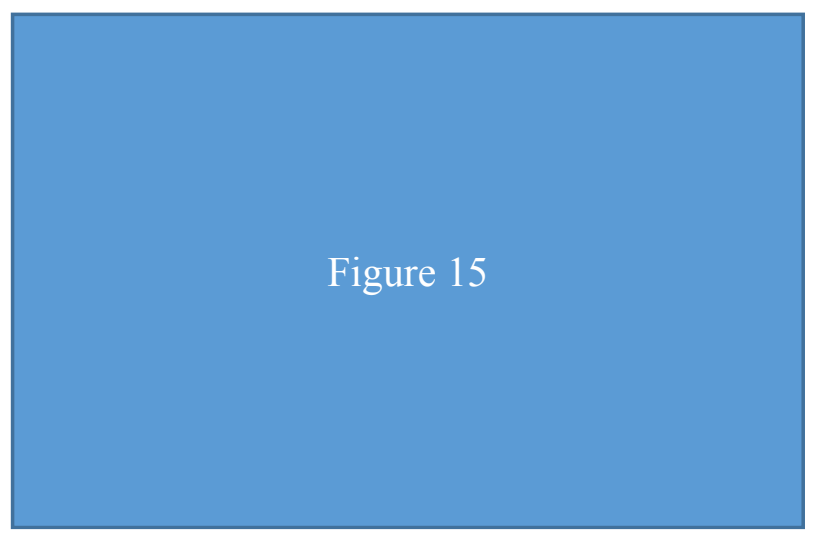


efficiency than other designs, without the omnidirectional design. The efficiency is increased $16.4 \%$ in the center of abdomen. This system gives the opportunity to be solved the huge problem of energy. Moreover such system can use more powerful ASICs in order to achieve better compression ratio. As a result, high resolution cameras can be used. The benefits of this design are not only in camera capsules but they can be used in robotic capsules that need more energy for their mechanical parts.

\subsection{Locomotion}

In the previous section, a description of internal locomotion systems has been presented. There is extensive research on capsules with an external locomotion system.

The method used for external locomotion of capsule is by the use of magnetic fields. Such systems have been introduced previously for use inside human's eye.

Magnetic locomotion systems have a lot of advantages. The most useful and critical advantage of that the system, unlike internal locomotion system, does not need a lot of components, just coils or permanent magnets. This means less space is needed. Such a system doesn't need actuation mechanisms and extra batteries for locomotion. In the design of an external locomotion system the use of magnetic coils or a permanent magnet are taken under consideration. The use of magnetic coils can provide better control of the capsule but permanent magnets can provide a stronger magnetic field in a more compact system and without the use of a power source.

It is obvious that the external locomotion technology has a lot of benefits and has started researching for a commercial product. In their design for the external magnetic coil they used a handheld magnet to generate the magnetic field for the capsule's locomotion.

The use of external locomotion in capsule endoscopy is a state of the art technology. There are also some drawbacks that should be solved. The equipment required for control of the capsule's locomotion is expensive and in some cases the patient must stay to the hospital to do the diagnosis. Furthermore the cost of such systems may be prohibited for use in small clinics or in doctor's offices. Although the advantages of these systems can provide accurate diagnosis and can perform better biopsy for patients. A hybrid system that can combine the advantages of both external and internal locomotion can achieve better results and solve the problem of locomotion.

\subsection{Image processing}

Image processing is another interesting research that helps WCEs. Image processing algorithms can be used to help the diagnosis process by providing detection of various gastrointestinal disorders. Algorithms for blood detection and Crohn's disease are already implemented. Both of these works provide a new way of detection on the invalid area.

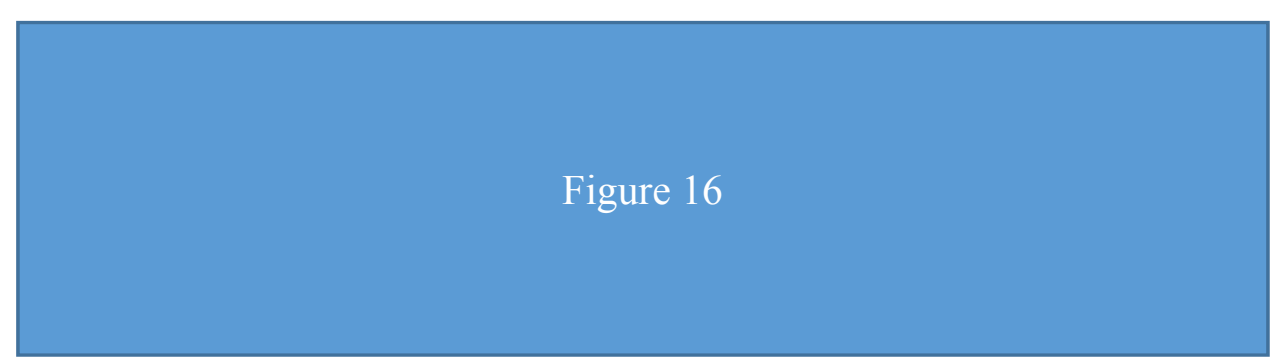


The detection algorithms utilize pixel by pixel comparison for detection. These new approaches are focused in the use of a cluster of pixels and not separate pixels. This eliminates the detection of some areas where in fact there is no bleeding or having Crohn's disease. In order to achieve better accuracy, machine learning methods such as Support Vector Machine (SVM) classifier was used. Figure 16 shows the results.

\section{$4 \quad$ Discussion and future outlooks}

Capsule endoscopy is a very interesting research area. There are different gastrointestinal environments due to various diagnoses need. These are relevant to different types of capsules with varying abilities. There are capsules with biosensors and the ability of external/internal locomotion for tissue manipulation, or camera capsules without locomotion and only a localization system.

Currently, extensive research efforts focused on the improvement of the limitations of the sub-systems in a WCE. It is clear that the resolution of the camera is too low for accurate diagnostic purposes. High resolution camera and probably 3D cameras can be used and it will change the point of view of the physician. Different placement of the cameras can give the ability to capture $360^{\circ}$ degrees of view and the gastrointestinal mapping can be achieved. The use of CMOS camera sensors and infrared LEDs, can capture a different view of the gastrointestinal (GI) and provide more useful data to the physicians. In the camera system, there are a lot of research tasks, such as camera lens and the ability of them to focus. Compression algorithms are also required for reduction of the transmitted data. The design of a low complexity algorithm with high compression ratio can reduce the energy required for wireless transmission.

In the area of sensors, the ability to construct smaller and more accurate sensors can provide better results for diagnosis. WCEs with all the required sensors are already in use, but WCEs with both camera and sensors has not been implemented yet. Also by the use of 3-axis acceleration sensor, the orientation of the pill can be determined. Such systems, which combine a camera with $360^{\circ}$ view and localization system, can synthesize a virtual map of the gastrointestinal (GI) with real images. In a system like this, the doctor/physician can "walk" through the virtual gastrointestinal (GI) with the use of software in a PC and provide better diagnostic results than current systems.

There are sub-systems in a WCE that play major role in the final result of the diagnosis such as the telemetry and the power supply. The use of a compression algorithm combined with a state of the art telemetry system can reduce the energy consumption and let the WCE to operate for more time in the gastrointestinal (GI). A major disadvantage of existing systems is that the energy of the battery is not enough to capture the entire gastrointestinal (GI) and sometimes the interesting area for diagnosis is not captured. The use of a wireless energy transfer system can provide the amount of energy required to operate until the end of gastrointestinal (GI). An efficient wireless energy transfer system can give more energy than a battery, but may require more space. The use of such a system can enhance the entire design of the capsule. More energy means more power for computations (compression algorithm) and more energy for the telemetry system. It is obvious that with more energy, the use of WCE for operation can become a reality. Live "streaming" of the camera and a bi-directional telemetry system can used for robotic capsules and allow doctors to perform operations to the gastrointestinal (GI) without the use of anesthesia and with less recovery time for patients. 
In future systems, different combination of various sub-systems into one capsule will be more to see. The abilities of capsule endoscopy are bound to increase and the diagnostic and surgical introduction is sure to become a reality. Their size will be decreased more and their use in smaller areas, of human body except of gastrointestinal (GI) track, will become feasible. Capsule endoscopy promises inexpensive and painless surgery without the use of anesthesia. The future of medical diagnosis by the use of WCE is bright.

\section{References}

Glukhovsky, A. (2003). 'Wireless capsule endoscopy', Sensor Review, Vol. 23, pp 128133

McCaffrey, C. O'Mathuna C., Twomey, K. (2008), 'Swallowable-Capsule Technology', Pervasive Computing, IEEE, Vol. 7 , No. 1, pp 23 - 29.

Cao H, Rao S, Tang SJ, Tibbals HF, Spechler S, Chiao JC., (2013) 'Batteryless implantable dual-sensor capsule for oesophageal reflux monitoring', Gastrointestinal Endoscopy, pp 649-653.

Given Image Ltd. [Online]. http://www.givenimage.com. [Accessed 27 March 2013].

Given Image Ltd. [Online]. http://www.givenimage.com/en-int/InnovativeSolutions/Capsule-Endoscopy/Pillcam-ESO/Pages/default.aspx. [Accessed 24 July 2013].

Given Image Ltd. [Online]. http://www.givenimage.com/en-int/InnovativeSolutions/Capsule-Endoscopy/Pillcam-COLON/Pages/default.aspx. . [Accessed 24 July 2013].

Liu X., Gu J., Xie Y., Xiong J., Qin W., (2012) 'A new approach to detecting ulcer and bleeding in Wireless capsule endoscopy images', IEEE-EMBS International Conference on Biomedical and Health Informatics (BHI), pp 737 - 740

Karargyris, A., Bourbakis, N., (2009) 'Identification of ulcers in Wireless Capsule Endoscopy videos', IEEE International Symposium on Biomedical Imaging: From Nano to Macro. June 28 2009-July 12009 , pp 554 - 557

Woods, S.P., Constandinou, T.G., (2013) 'Wireless Capsule Endoscope for Targeted Drug Delivery: Mechanics and Design Considerations', IEEE Transactions on Biomedical Engineering, Vol. 60, No. 4, pp 945 - 953.

Kong S.D, Choi C., Khamwannah, J., Jin S. (2013), 'Magnetically Vectored Delivery of Cancer Drug Using Remotely On-Off Switchable NanoCapsules', IEEE Transactions on Magnetics, Vol.49, No. 1, pp 349 - 352.

Yim S., Goyal, K., Sitti, M., (2013) 'Magnetically Actuated Soft Capsule With the Multimodal Drug Release Function', IEEE/ASME Transactions on Mechatronics, Vol. 18 , No. 4, pp 1413 - 1418.

Htwe T. M., Poh C. K., Li L., Liu J., Ong E. H., Ho K. Y., (2011) 'Vision-based techniques for efficient Wireless Capsule Endoscopy examination', Defense Science Research Conference and Expo (DSR), pp $1-4$.

RF Co.,Ltd., [Online]. Available: http://www.rfsystemlab.com/en/sayaka /index.html. [Accessed 3 November 2012].

Olympus, [Online]. 'EndoCapsule System EC-1', http://www.olympuseuropa.com/medical/en/medical_systems/products_services/product_details/pro duct_details_17984.jsp. [Accessed 3 November 2012]. 
Gonzalez-Guillaumin, J., Sadowski, D., Kaler, K., and Mintchev, M. (2007) 'Ingestible capsule for impedance and $\mathrm{pH}$ monitoring in the esophagus', IEEE Trans. Biomed. Eng., Vol.54, Is.12, pp2231-2236.

McKenzie J. and Osgood D., (2004) 'Validation of a new telemetric core temperature monitor', J. Therm. Biol., Vol. 29, No. 7-8, pp 605-611.

BMedical Pty Ltd, [Online].: http://www.bmedical.com.au/shop/activity-heatresearch/vitalsense.htm [Accessed 3 April 2013]

Johannessen E. A., Wang L., Cui L., Tang T. B., Ahmadian M., Astaras A. Reid, S. W. J. Yam, P. S., Murray A. F., Flynn B.W., Beaumont S. P., Cumming D. R. S., and Cooper J. M. (2004) 'Implementation of multichannel sensors for remote biomedical measurements in a microsystems format', IEEE Trans. Biomed. Eng., Vol. 51, pp 525-535.

Seo S. W., Han S., Seo J. H., Choi W. B. and Sung M. Y., (2010) 'Liquid Lens Module with Wide Field-of-View and Variable Focal Length', Electronic Materials Letters, Vol. 6, No. 4, pp 141-144.

Cavallotti C., Merlino P.,Vatteroni M., Valdastri P., Abramo A., Menciassi A., Dario P., (2011) 'An FPGA-based versatile development system for endoscopic capsule design optimization', Sensors and Actuators A, Vol. 172, No. 1, pp 301- 307.

Xie X., Li G., Chen X., Li X., and Wang Z., (2006) 'A Low-Power Digital IC Design Inside the Wireless Endoscopic Capsule', IEEE Journal on Solid-State Circuits, Vol. 41, No. 11, pp $2390-2400$.

Thonı J., Radiom S., Turgis D., Carta R., Gielen G., Puers R., (2009) 'Design of a 2 Mbps FSK near-field transmitter for wireless capsule endoscopy', Sensors and Actuators A, Vol. 156, No. 1, pp 43-48.

Carta, R., Puers, R., (2011) 'Wireless power and data transmission for robotic capsule endoscopes', 18th IEEE Symposium on Communications and Vehicular Technology in the Benelux (SCVT), pp 1-6.

Moglia A., Menciassi A., Dario P., and Cuschieri A., (2009) 'Capsule endoscopy: progress update and challenges ahead', Nat. Rev. Gastroenterol. Hepatol., Vol. 6, pp 353-362.

Quirini M., Webster R. J., Menciassi A., and Dario P., (2007) 'Design of a Pill-Sized 12legged Endoscopic Capsule Robot', IEEE International Conference on Robotics and Automation, Roma, pp 1856 - 1862.

Lenaerts B. and Puers R., (2007) 'An inductive power link for a wireless endoscope', Biosensors and Bioelectronics, Vol. 22, pp 1390-1395.

Karagozler M. E., Cheung E., Kwon J., and Sitti M., (2006) 'Miniature Endoscopic Capsule Robot using Biomimetic Micro-Patterned Adhesives', The First IEEE/RAS-EMBS International Conference on Biomedical Robotics and Biomechatronics, pp $105-111$.

Olympus [Online]. http://www.olympusamerica.com/msg_section/endocapsule/. [Accessed 8 June 2013].

Ciuti G., Menciassi A. and Dario P. (2011) 'Capsule Endoscopy: From Current Achievements to Open Challenges', IEEE Reviews on Biomedical Engineering, Vol. 4, pp $59-72$.

Turcza P. and Duplaga M., (2013) 'Hardware-Efficient Low-Power Image Processing System for Wireless Capsule Endoscopy', IEEE Journal on Biomedical and Health Informatics, Vol. 17, No. 6, pp 1046 - 1056.

Shirsat T. G. and Bairagi V. K., (2013) 'Lossless Medical Image Compression by Integer Wavelet and Predictive Coding', ISRN Biomedical Engineering, vol. 2013, Article ID 832527, 6 pages. 
Gao Y., Cheng S. J., Toh D., Kwok Y. S., Tan K. B., Chen X., Mok W., Win H., Zhao B., Diao S., Alper C., Zheng Y., Sun S., Je M., and Heng C. (2012) 'An Asymmetrical QPSK/OOK Transceiver SoC and 15:1 JPEG Encoder IC for Multifunction Wireless Capsule Endoscopy', IEEE Asian Solid-State Circuits Conference, pp $341-344$.

Kasun M.S. Thotahewa, Jean-Michel Redouté, and Mehmet R. Yuce, (2013) 'Electromagnetic power absorption of the human abdomen from IR-UWB based wireless capsule endoscopy devices', 2013 IEEE International Conference on Ultra-Wideband (ICUWB), pp $79-84$.

Pourhomayoun M., Jin Z., and Fowler M., (2013) 'Accurate Localization of In-Body Medical Implants Based on Spatial Sparsity', IEEE Transactions on Biomedical Engineering, online.

Sun T. J., Xie X., Li G. L., Gu Y. K., Deng Y. D. and Wang Z.H., (2012) 'Integrated omnidirectional wireless power receiving circuit for wireless endoscopy', Electronics Letters, Vol. 48 No. 15, pp $907-908$.

Ma J., Tillo T., Zhang B., Wang Z., Lim E. G., (2013) 'Novel training and comparison method for blood detection in wireless capsule endoscopy images', 7th International Symposium on Medical Information and Communication Technology (ISMICT), pp 56-60.

Jebarani, W.S.L., Daisy, V.J., (2013) 'Assessment of Crohn's disease lesions in Wireless Capsule Endoscopy images using SVM based classification', 2013 International Conference on Signal Processing Image Processing \& Pattern Recognition (ICSIPR), pp $303-307$.

\section{Bibliography}

Ergeneman O., Dogangil G., Kummer M. P., Abbott J. J., Nazeeruddin M. K. and Nelson B. J. (2008) 'A magnetically controlled wireless optical oxygen sensor for intraocular measurements', IEEE Sen. J. Am. Chem. Soc., Vol. 8, No. 1, pp 2937.

Volke F., Keller J., Schneider A., Gerber J., Reimann-Zawadzki M., Rabinovitz B., Mosse C. and Swain P. (2008) 'In-vivo remote manipulation of modified capsule endoscopes using an external magnetic field', Gastrointestinal Endoscopy, Vol. 67, No. 5, pp AB121-AB122.

Yim S. and Sitti M., (2012) 'Design and Rolling Locomotion of a Magnetically Actuated Soft Capsule Endoscope', IEEE Transactions on Robotics, Vol. 28, No. 1, pp 183-194.

Choi H., Jeong S., Lee C., Ko Y., Ko S. Y., Park J. O. and Park S., (2011) 'Biomimetic swimming mini-robots using electro-magnetic actuation (EMA) system', 12th International Conference on Control, Automation and Systems (ICCAS), pp $1923-1926$.

Wang X., Meng, M.Q.-H. and Chen X., (2010) 'A locomotion mechanism with external magnetic guidance for active capsule endoscope', 2010 IEEE Annual International Conference of Engineering in Medicine and Biology Society (EMBC), , pp $4375-4378$. 


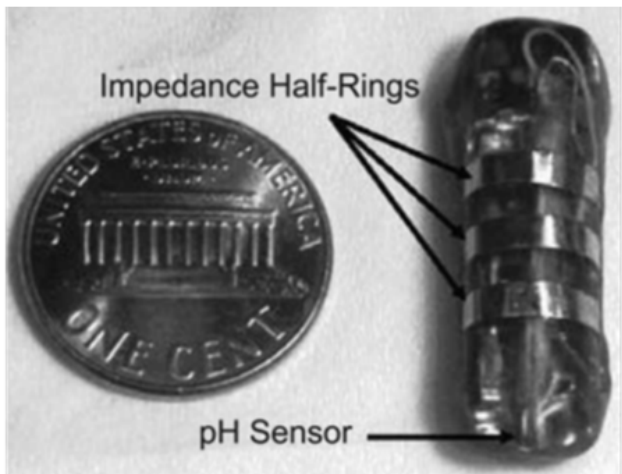

Figure 1 Prototype of the $\mathrm{pH}$-sensing capsule, the $\mathrm{pH}$ sensor and the half-rings for impedance sensing can observed

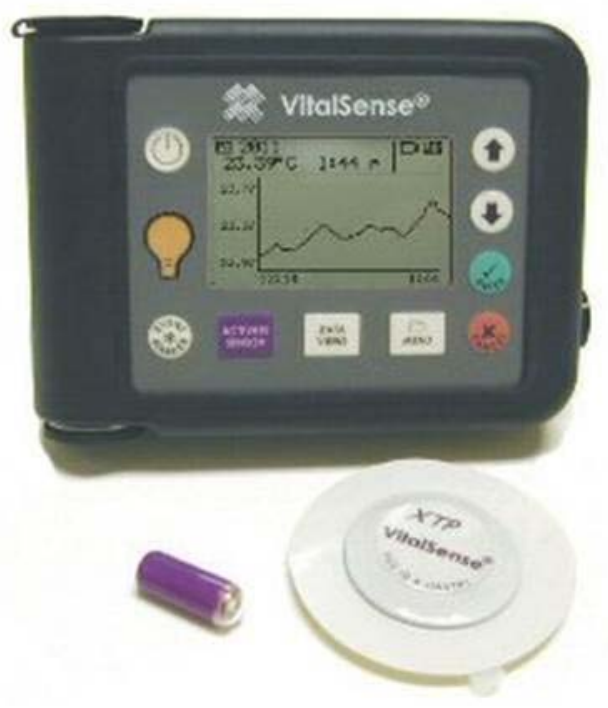

Figure 2 VitalSense pill system 

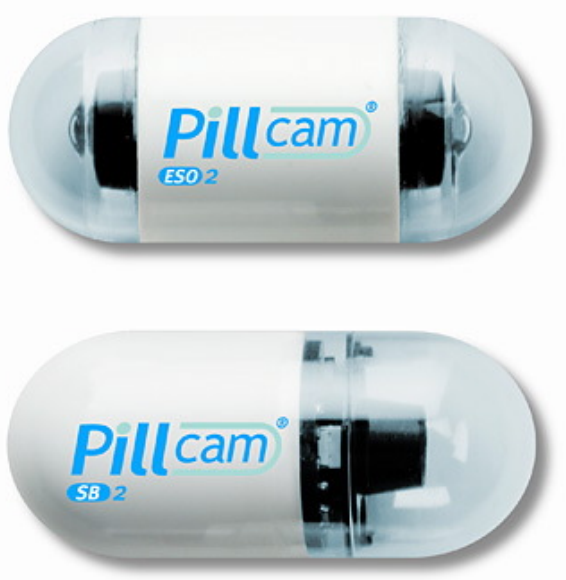

Figure 3 PillCam horizontal placement of the camera

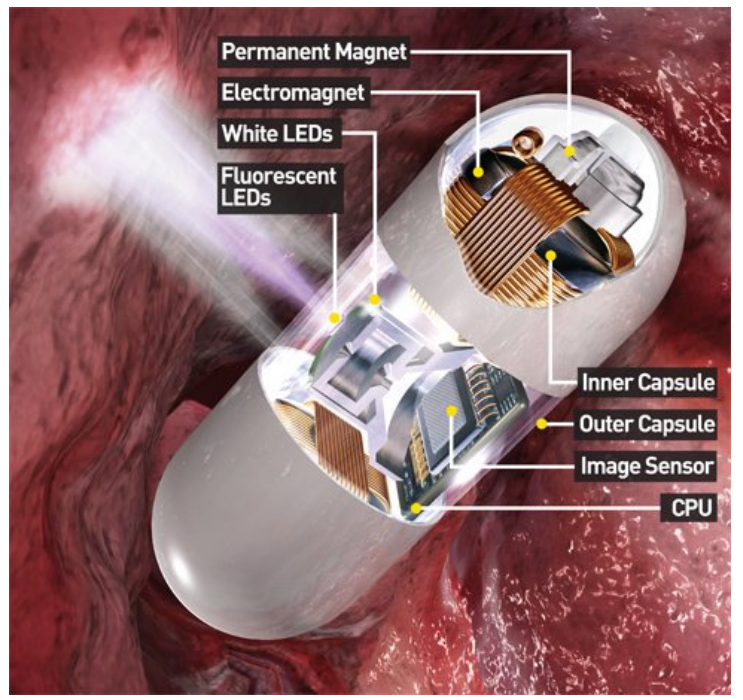

Figure 4 SAYAKA pill vertical placement of the camera 


\begin{tabular}{|c|c|c|c|c|}
\hline Capsule/Pill & Size mm & Vision & Resolution & Company/University \\
\hline PillCam & $27 \times 11$ & CMOS & $256 \times 256$ & Given Image \\
\hline Norika & $23 \times 9$ & CCD & $320 \times 320$ & RF System Lab, JP \\
\hline Endoscope & $30 \times 11$ & CMOS & - & Uni Kyungpook, KR \\
\hline Endocapsule & $26 \times 11$ & CCD & 2 Mpixel & Olympus \\
\hline IVP & $23 \times 11$ & CMOS & - & IMS, DE \\
\hline SAYAKA & NA & CMOS & 2 Mpixel & RF System Lab, JP \\
\hline MiRo & NA & CMOS & $320 \times 320$ & - \\
\hline
\end{tabular}

Table 1Existing WCE with camera and their characteristics

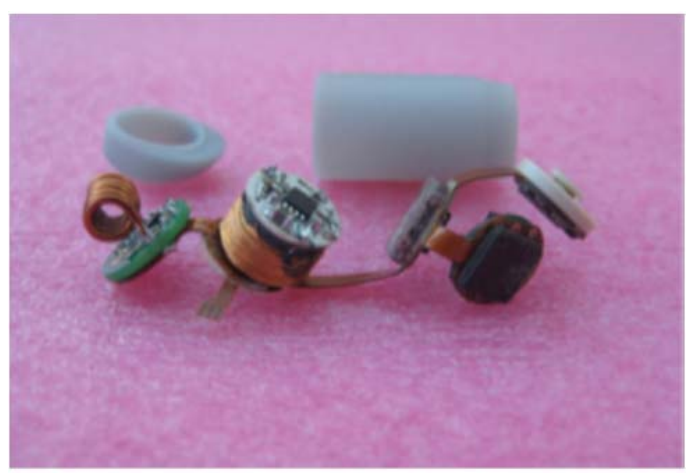

Figure 6 Miniaturized prototype

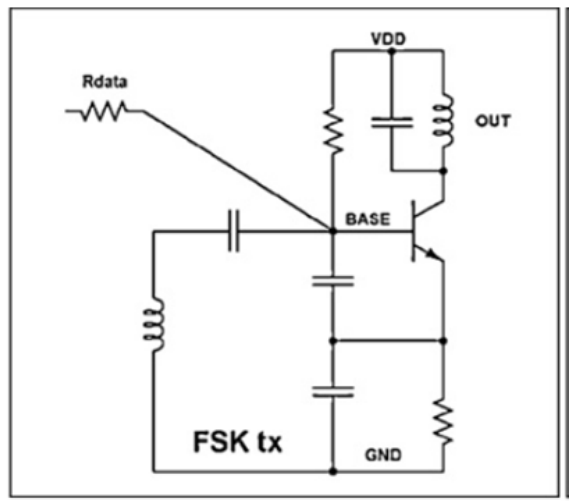

Figure 5 Wireless transmitter of $2 \mathrm{Mbps}$ and $2 m W$ 


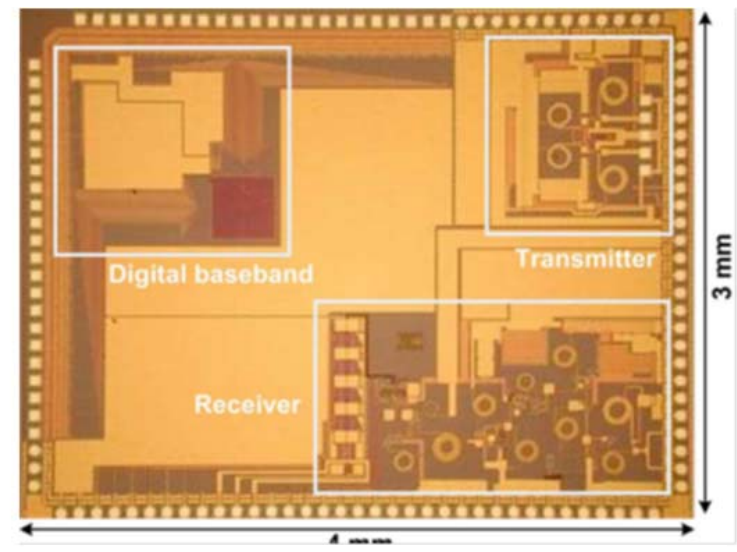

Figure 7 Die photo of the transceiver system

\begin{tabular}{|c|c|c|c|}
\hline & $\begin{array}{c}\text { Power } \\
\text { requirements } \\
{[\mathrm{mW}]}\end{array}$ & $\begin{array}{l}\text { Freq. } \\
\text { [MHz] }\end{array}$ & $\begin{array}{c}\text { Data } \\
\text { rate } \\
\text { [Mbps] }\end{array}$ \\
\hline Shen (2005) & 4 mW@1.85V & 416 & 2 \\
\hline Chi (2007) & $7.9 \mathrm{~mW} @ 2.5 \mathrm{~V}$ & 2400 & 1 \\
\hline Itoh (2006) & 1.4 mW@2V & 20 & 2.5 \\
\hline Liu (2007) & $19.5 \mathrm{mW@1.5 \textrm {V }}$ & 400 & 1.5 \\
\hline Hancock (2007) & 9.7 mW@1.8V & $1.35-1.75 \mathrm{GHz}$ & 2 \\
\hline Turis (2007) & $6 \underline{\mathrm{mW} @ 1.7 \mathrm{~V}}$ & 120 & 1 \\
\hline 2Mbps-2mW TX (2008) & 2 mW@1.9V & 144 & 2 \\
\hline UHF Transmitter(2010) & 3.9mW@,2.5-3.3V & 400 & 3 \\
\hline $\begin{array}{l}\text { A Low-Power } 13.56 \\
\operatorname{MHz}(2012)\end{array}$ & 0.594mW@1.8V & 13.56 & - \\
\hline $\begin{array}{l}2 \text { Mbps FSK near-field } \\
\text { transmitter(2009) }\end{array}$ & 2mW@1.8V & 144 & 2 \\
\hline $\begin{array}{l}\text { Wireless power and data } \\
\text { transmission(2011) }\end{array}$ & $5 \mathrm{~mW}$ & 333 & 2 \\
\hline $\begin{array}{l}\text { Low-Power Ultra wideband } \\
\text { Wireless Telemetry(2011) }\end{array}$ & $0.35 \mathrm{~mW}$ & $3-5 \mathrm{GHz}$ & 10 \\
\hline $\begin{array}{l}\text { A Low-Power, High Data- } \\
\text { Rate } \\
\text { CMOS ASK(2012) }\end{array}$ & 7.2mW@1.8V & $902-928$ & 15 \\
\hline
\end{tabular}

Table 2 Comparison table of existing wireless systems for capsule endoscopy 


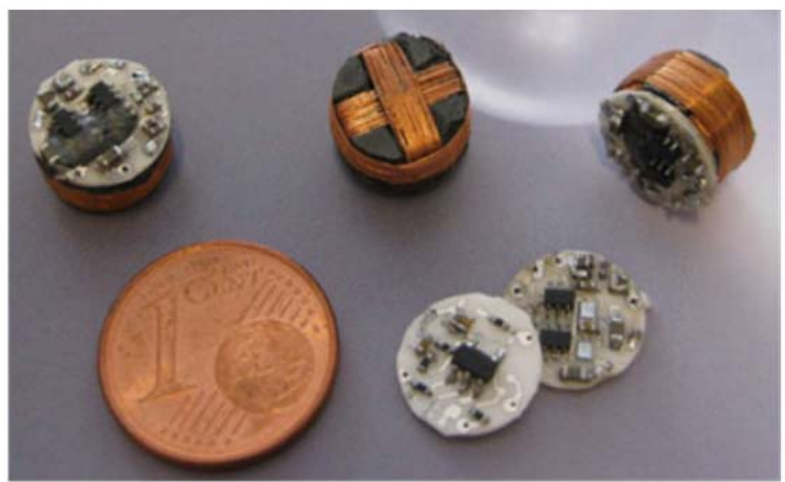

Figure 83D coils of an energy harvesting system compared with the size of 1-cent coil

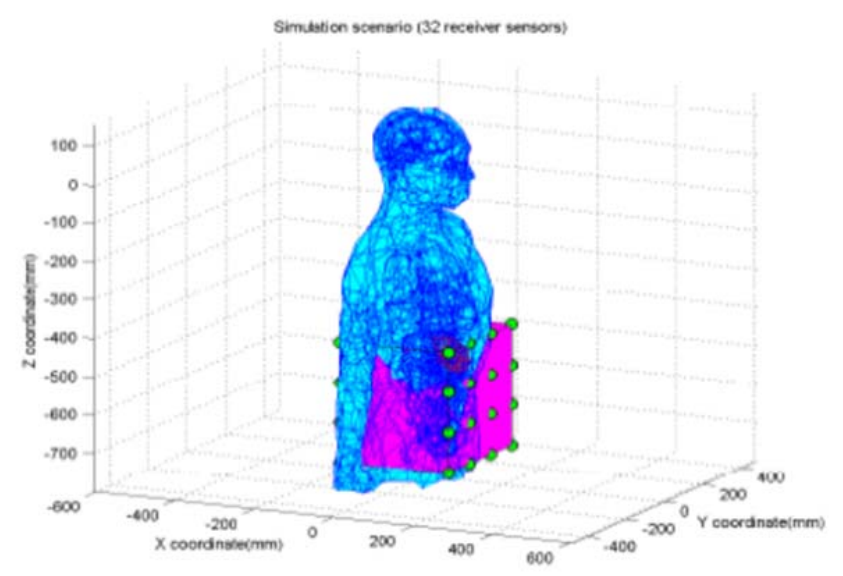

Figure 9 Simulation of 32 sensor localization system

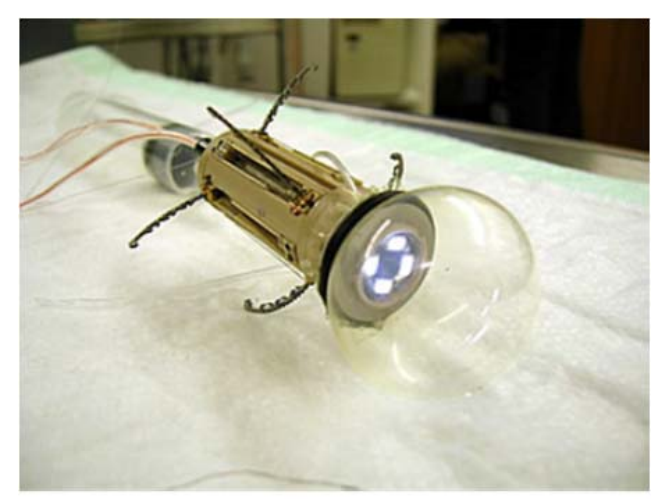

Figure 10 Capsule with legged locomotion 


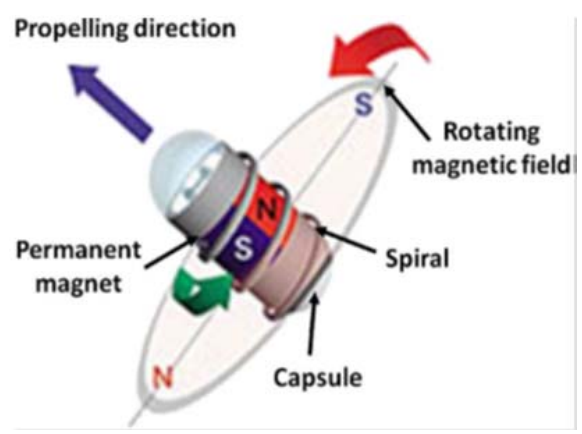

Figure 11 Olympus Inc. magnetic locomotion capsule concept

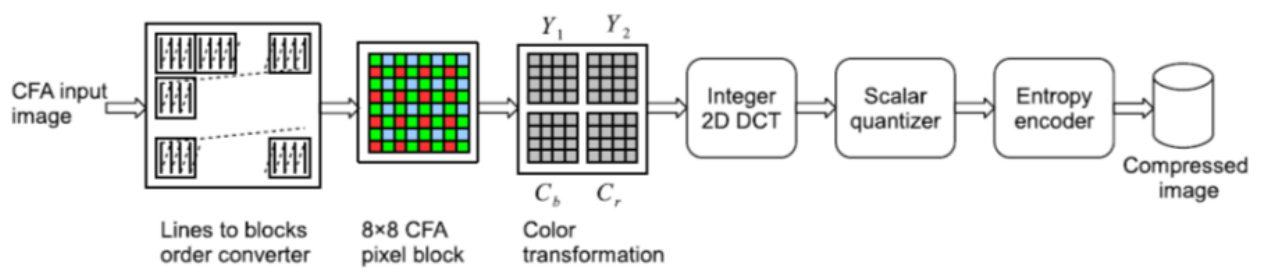

Figure 12 Block diagram of the compression algorithm

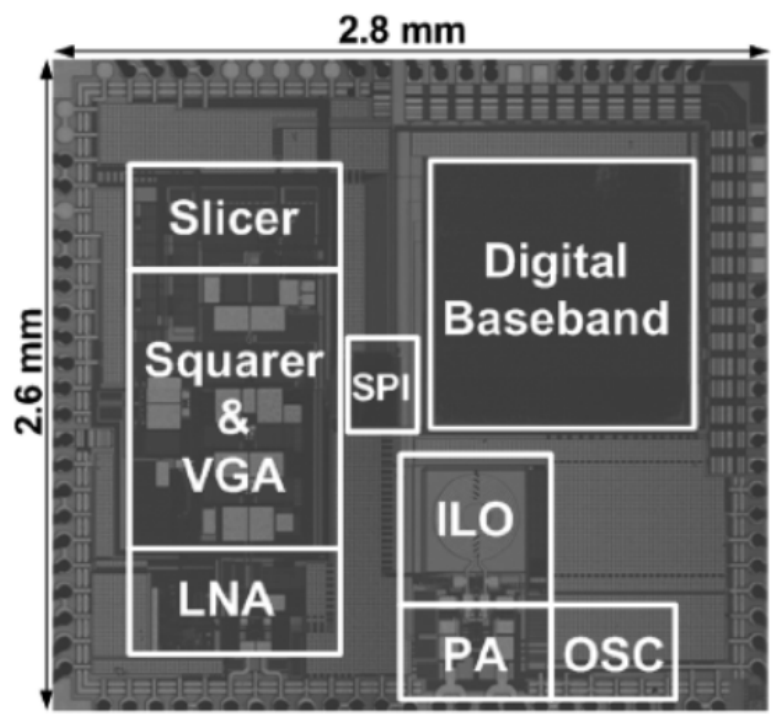

Figure 13 Photograph of the entire IC with individual parts 


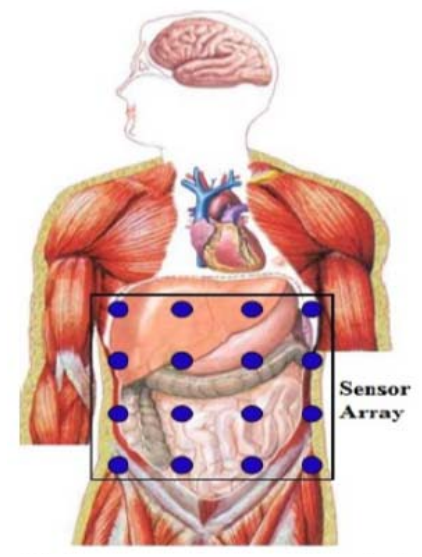

Figure 14 Sensors arrangement for capsule localization

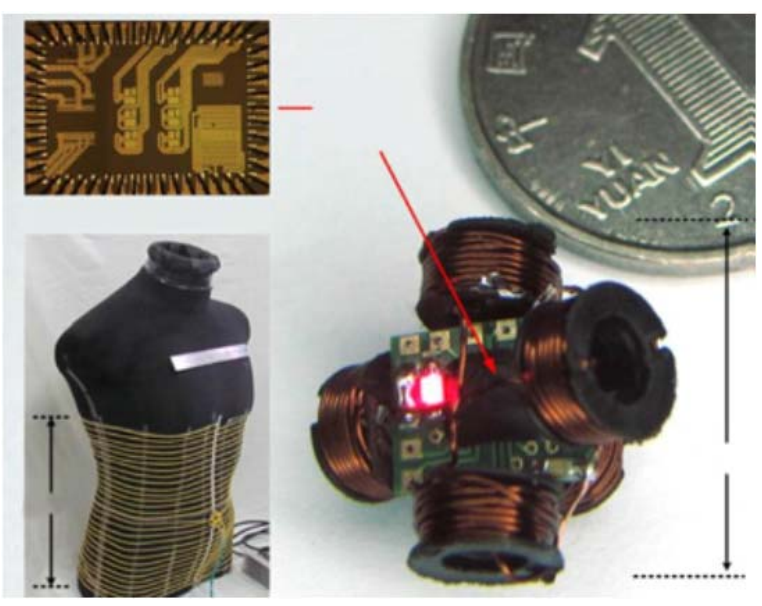

Figure 15 Design of the omnidirectional energy receiver

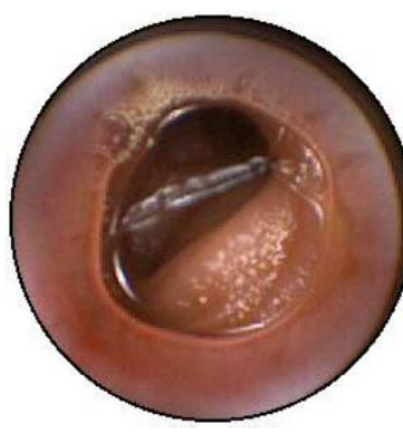

(a)

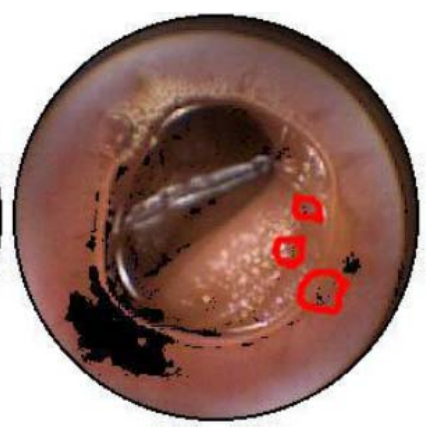

(b)

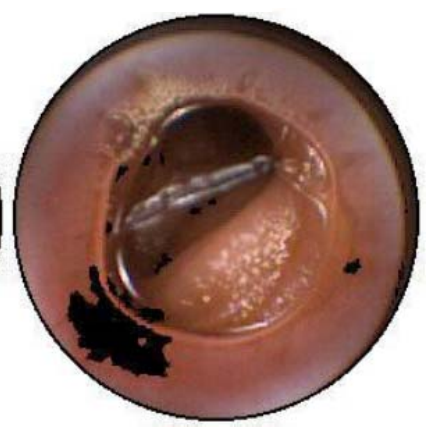

(c)

Figure 16 (a) There is the uncompressed-unprocessed image, (b) the blood detection made without the use of proposed algorithm, and (c) the machine learning based algorithm is used. 